\title{
The impact of the Trauma Triage App on pre-hospital trauma triage: design and protocol of the stepped-wedge, cluster- randomized TESLA trial
}

Rogier van der Sluijs ${ }^{1,2,3^{*}}$ (D) Audrey A. A. Fiddelers ${ }^{3}$, Job F. Waalwijk ${ }^{1,2,3}$, Johannes B. Reitsma ${ }^{4}$, Miranda J. Dirx ${ }^{3}$, Dennis den Hartog ${ }^{5}$, Silvia M. A. A. Evers ${ }^{6}$, J. Carel Goslings, ${ }^{7,8}$, W. Margreet Hoogeveen ${ }^{9}$, Koen W. Lansink ${ }^{10}$, Luke P. H. Leenen', Mark van Heiji ${ }^{2,11}$ and Martijn Poeze ${ }^{1,3}$

\begin{abstract}
Background: Field triage of trauma patients is crucial to get the right patient to the right hospital within a particular time frame. Minimization of undertriage, overtriage, and interhospital transfer rates could substantially reduce mortality rates, life-long disabilities, and costs. Identification of patients in need of specialized trauma care is predominantly based on the judgment of Emergency Medical Services professionals and a pre-hospital triage protocol. The Trauma Triage App is a smartphone application that includes a prediction model to aid Emergency Medical Services professionals in the identification of patients in need of specialized trauma care. The aim of this trial is to assess the impact of this new digital approach to field triage on the primary endpoint undertriage.

Methods: The Trauma triage using Supervised Learning Algorithms (TESLA) trial is a stepped-wedge clusterrandomized controlled trial with eight clusters defined as Emergency Medical Services regions. These clusters are an integral part of five inclusive trauma regions. Injured patients, evaluated on-scene by an Emergency Medical Services professional, suspected of moderate to severe injuries, will be assessed for eligibility. This unidirectional crossover trial will start with a baseline period in which the default pre-hospital triage protocol is used, after which all clusters gradually implement the Trauma Triage App as an add-on to the existing triage protocol. The primary endpoint is undertriage on patient and cluster level and is defined as the transportation of a severely injured patient (Injury Severity Score $\geq 16$ ) to a lower-level trauma center. Secondary endpoints include overtriage, hospital resource use, and a cost-utility analysis.

Discussion: The TESLA trial will assess the impact of the Trauma Triage App in clinical practice. This novel approach to field triage will give new and previously undiscovered insights into several isolated components of the diagnostic strategy to get the right trauma patient to the right hospital. The stepped-wedge design allows for within and between cluster comparisons.

(Continued on next page)
\end{abstract}

\footnotetext{
*Correspondence: r.vandersluijs@icloud.com; r.vandersluijs-3@umcutrecht.nl

'Department of Surgery, Maastricht University Medical Center, Maastricht,

The Netherlands

${ }^{2}$ Department of Surgery, Utrecht University Medical Center, Utrecht, The

Netherlands

Full list of author information is available at the end of the article
}

(c) The Author(s). 2020 Open Access This article is licensed under a Creative Commons Attribution 4.0 International License, which permits use, sharing, adaptation, distribution and reproduction in any medium or format, as long as you give appropriate credit to the original author(s) and the source, provide a link to the Creative Commons licence, and indicate if changes were made. The images or other third party material in this article are included in the article's Creative Commons licence, unless indicated otherwise in a credit line to the material. If material is not included in the article's Creative Commons licence and your intended use is not permitted by statutory regulation or exceeds the permitted use, you will need to obtain permission directly from the copyright holder. To view a copy of this licence, visit http://creativecommons.org/licenses/by/4.0/. 


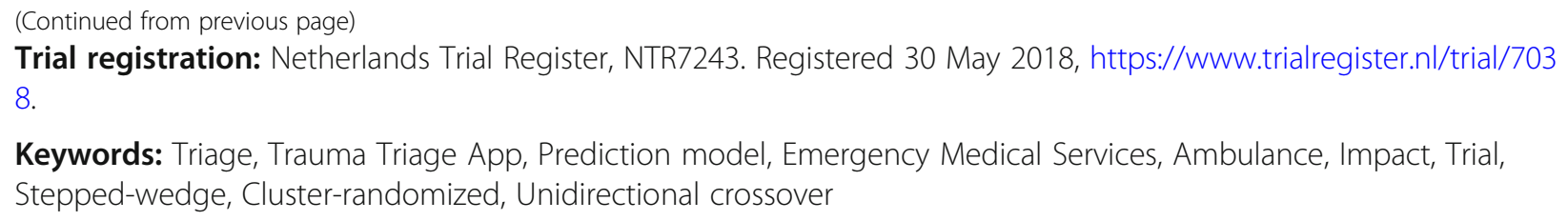

\section{Background}

Pre-hospital trauma triage is crucial to match an injured patient to the optimal definitive care facility [1]. Erroneously transporting a patient requiring specialized trauma care to a lower-level trauma center could lead to a delay in definitive care and is associated with higher mortality and morbidity rates [2]. Conversely, transporting a patient not in need of specialized trauma care to a higher-level trauma center results in extra costs and overutilization of resources [3]. These key metrics to evaluate the quality of field triage in trauma systems are termed undertriage and overtriage, respectively [1]. The Dutch National Health Care Institute guidelines state that a maximum of $10 \%$ undertriage is acceptable in the Netherlands [4]. The mean undertriage across all inclusive trauma regions in the Netherlands was $31.4 \%$ in 2016 [5].

The first step of the multicomponent strategy to determine the optimal receiving facility is to identify patients in need of specialized trauma care. This is performed by Emergency Medical Services (EMS) professionals onscene and is influenced by both the pre-hospital triage protocol and the judgment of the EMS professional. The 8th version of the National Protocol of Ambulance Services (NPAS; in Dutch Landelijk Protocol Ambulancezorg) is currently used by all EMSs in the Netherlands as the primary pre-hospital triage protocol. A recent study reported that strict adherence to the criteria of the NPAS would have led to an undertriage rate of $63.8 \%$, with an overtriage rate of $7.3 \%$ in one inclusive trauma region [6]. Moreover, a systematic review did not identify any pre-hospital triage protocol that by itself complied with the target of $10 \%$ undertriage [7].

The Trauma Triage App (TTApp), a smartphone and tablet application that incorporates a prediction model, was recently developed to identify patients in need of specialized trauma care. The main function of the TTApp is to predict an individual patient's probability of being severely injured. An advice regarding whether a patient requires specialized trauma care is then generated based on a pre-defined threshold probability. This novel approach to trauma triage was externally validated retrospectively in 6859 patients from a different EMS and was able to retain a c-statistic of 0.83 with proper calibration [8]. An undertriage rate of $11.2 \%$ with a combined overtriage rate of less than $50 \%$ can hereby be achieved, depending on the threshold probability.
Notwithstanding these promising validation results, the impact of the use of the TTApp in daily practice remains to be established. The TESLA (Trauma triagE using Supervised Learning Algorithms) stepped-wedge cluster-randomized trial was designed to evaluate whether the availability of the TTApp during field triage indeed leads to a decrease in undertriage, while preserving acceptable overtriage rates.

\section{Methods/design Study design}

The TESLA trial is a prospective, stepped-wedge clusterrandomized trial (SW-CRT). In a SW-CRT, clusters are randomized into allocation sequences. These sequences all start with one or more periods under the control condition, followed by the remaining periods in which the intervention is implemented. In this trial, the participating EMS regions (the clusters) will be randomized upfront to determine the period after which two paired clusters will switch to the intervention condition. Clusters within the same inclusive trauma region were paired to ease implementation in practice. Randomization of allocation sequence was completed using computer random number generator on the primary research site (RvdS) prior to the recruitment of participants. Simple randomization was preferred to other techniques as the number of clusters was small and because there was no need to balance the influence of covariates. No methods to conceal the allocation sequence nor blinding was applied on either patient or cluster level. Our aim is to include 1920 consecutive severely injured patients in five steps, each with a duration of 4 months (details about the sample size below). All clusters will start with one or more steps of usual care (the NPAS). At the end of each step, two clusters will switch from the NPAS to the intervention condition (the TTApp used as an add-on to the NPAS). Key study design features are shown in Fig. 1.

\section{Participating regions}

Eight out of 25 EMSs (the clusters) in the Netherlands, with approximately equal patient volumes, were selected to participate in this trial (Fig. 2). A combination of EMSs with heterogeneous service areas (i.e., urban, suburban, and rural) was selected to improve the generalizability of the results. All participating EMS 


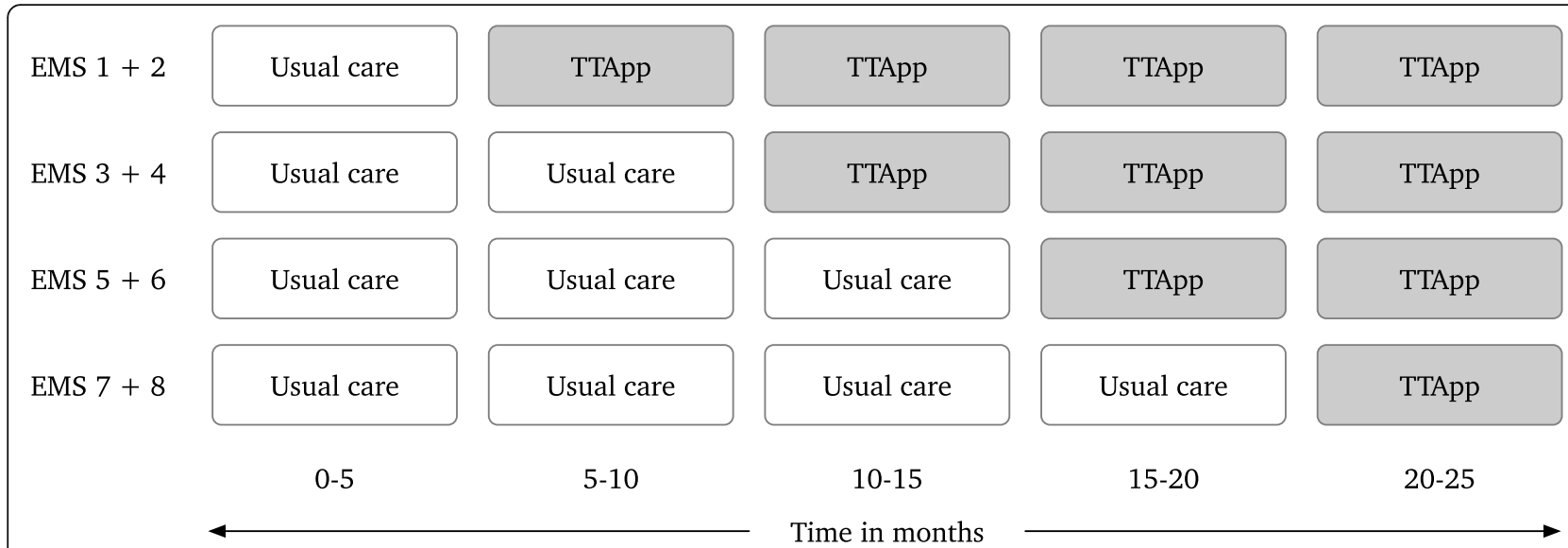

Fig. 1 The stepped-wedge design of the TESLA-trial. Abbreviations: EMS, Emergency Medical Service; TTApp, Trauma Triage App

regions take part in the Pre-hospital Trauma Triage Research Collaborative. These EMSs are an integral part of five distinct inclusive trauma regions and cover urban, suburban, and rural areas. All 37 hospitals with a trauma-receiving emergency department within these five distinct inclusive trauma regions participate in the collection of relevant patient outcomes. All hospitals with an emergency department within these regions are designated a level of care being either one, two, or three. Level one is considered a higher-level trauma center, whereas both level two and three trauma centers are acknowledged as lower-level trauma centers.

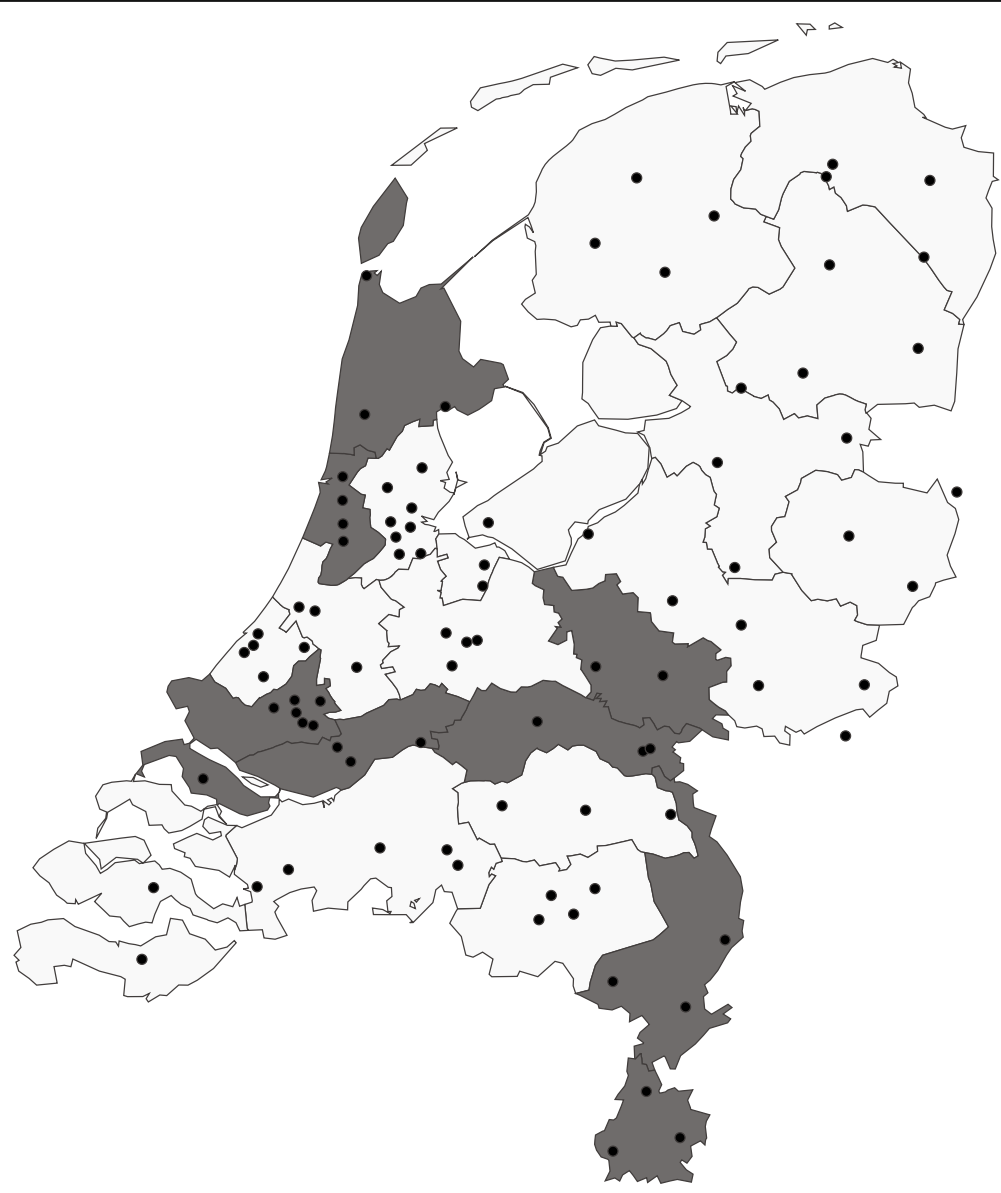

Fig. 2 Service regions of the participating Emergency Medical Services 


\section{Study population}

All patients, 18 years of age or older, evaluated on-scene by an EMS professional, suspected of moderate to severe injuries, defined as an Injury Severity Score (ISS) $\geq 9$, will be assessed for eligibility. Patients transported to a hospital outside of the participating trauma regions will be excluded. Patients that are dead on arrival at the initial receiving emergency department will also be excluded.

\section{Pre-hospital trauma triage tools \\ Usual care: National Protocol of Ambulance Services}

No adjustments to daily practice of EMS professionals will be introduced prior to the switch to the intervention period. Assessment of injury severity is often a two-step process consisting of the evaluation of the pre-hospital trauma triage protocol and the final judgment of the EMS professional. The 8th version of the NPAS is currently used by all participating EMSs. This protocol is a flowchart that consists of multiple criteria to identify patients in need of specialized trauma care. If a patient fulfills one of the criteria in Table 1, the EMS professional is advised to transport the patient to a higher-level trauma center. In daily practice, it is not obligatory to adhere to the protocol or to report its advice.

\section{Intervention: Trauma Triage App}

The TTApp is a smartphone and tablet application for both Android and iOS operating systems (Fig. 3). The application is a practical and quick-to-use questionnaire consisting of six questions that collect the required predictor values of the prediction model incorporated in the TTApp (Table 2). Four additional questions are added: the judgment of the EMS professional prior to the questionnaire, the judgment of the EMS professional after the advice returned by the prediction model, the transportation destination while displaying a map with

Table 1 Higher-level trauma center criteria of the National Protocol of Ambulance Services

ABC-unstable during evaluation on-scene
Revised trauma score $<11$
Deteriorating Glasgow Coma Scale
Glasgow Coma Scale $<9$
Flail chest
Amputation proximal to wrist or ankle
Two or more fractures of the femur and/or humerus
Penetrating injury of head, thorax, or abdomen
Unstable pelvic fracture
Body temperature $<32^{\circ} \mathrm{C}$
Neurologic deficit of one or more extremities
Anisocoria

Revised trauma score $<11$

Deteriorating Glasgow Coma Scale

Glasgow Coma Scale $<9$

Two or more fractures of the femur and/or humerus

Penetrating injury of head, thorax, or abdomen

(a) pelvic fracture

Anisocoria distances to nearby hospitals, and when applicable, a screen to specify reasons to bypass the preferred hospital. The incorporated prediction model calculates the probability that a patient is severely injured. An advice whether to transport a patient to a higher-level trauma center or not is generated based on a pre-defined threshold probability. This threshold determines the sensitivity and specificity of the prediction model. Filling out the questionnaire takes approximately $30-45 \mathrm{~s}$ and should be performed on-scene by EMS professionals.

Decision-making about whether to transport a patient to a higher- or lower-level trauma center will be carried out similarly to usual care, with the exception of the availability of the TTApp prediction and recommendations linked to that prediction. Also, alike the NPAS, the TTApp is a decision-support system that can be overruled by EMS professional judgment. Implementation of the TTApp will likely lead to the transportation of more (severely) injured patients to higher-level trauma centers, thus reducing undertriage. This might lead to a slight increase in overtriage.

The TTApp will be introduced to all EMSs in a systematic manner at the end of the baseline period. A presentation will be provided to all EMS professionals which teaches the rationale behind pre-hospital triage and the study protocol. An electronic-learning will be made available that will demonstrate the use of the TTApp. The application will subsequently be made available on the proprietary devices of the participating EMS. Additional teaching sessions will be organized for teams of ambulance professionals during the intervention phase in each region.

\section{Data collection}

Pre-hospital data of trauma patients is routinely collected by EMS professionals through digital run-reports. Extra variables, mainly answers to the questionnaire and usage information, will be collected by the TTApp in the intervention period. Clinical data, including all relevant patient outcomes, of all patients admitted to one of the participating hospitals will be collected through the trauma registries of each inclusive trauma region. Injury Severity Scores will be routinely calculated within 30 days after the date of injury by trained trauma registrars for all admitted patients and those who die in the emergency department. All other included patients are assumed to have minor or moderate injuries (ISS < 16). This assumption was validated for all patients discharged from the emergency department in a previous study [6]. Hospital data and pre-hospital data will be anonymized first, and then linked using a combined deterministic and probabilistic linkage scheme. This anonymized linkage approach was validated to be both highly sensitive 

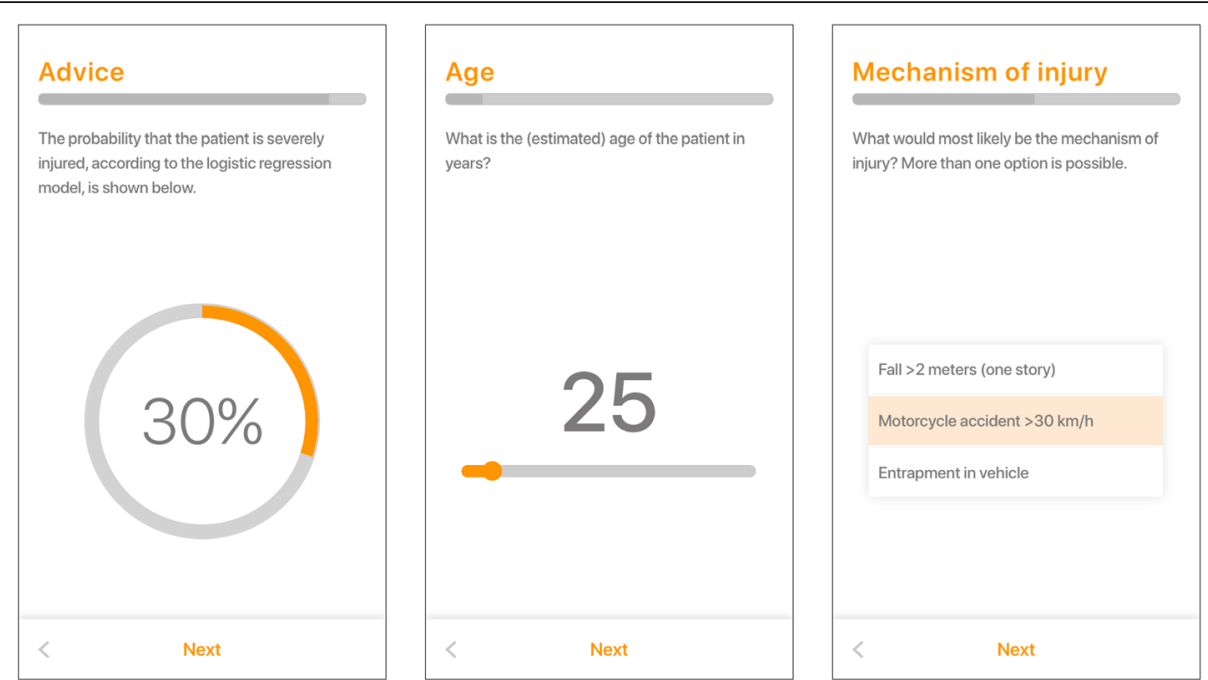

Fig. 3 The Trauma Triage App. A sample of screens from the Trauma Triage App. Left: the generated score indicating the probability that a patient might be severely injured based on all predictors. Middle: an input field requesting the age of the patient in years. Right: an input field requesting the mechanism of injury

and specific in prior research [6]. The final dataset will be accessible by JFW, MvH, and MP.

\section{Patient safety}

The TTApp is a diagnostic intervention aimed at EMS professionals. Regular care by EMS professionals should not be impacted by the TTApp. The prediction model is a decision support tool that-alike the NPAS-can be overruled by EMS professionals. This model is more sensitive and less specific compared to the NPAS, meaning that severely injured patients have a higher chance to get an advice for transportation to a higher-level trauma center. It will not be mandatory for EMS professionals to use the TTApp during the intervention period.

\section{Primary outcome}

Primary endpoint of the study is undertriage, defined as the transportation of a severely injured patient (ISS $\geq$ 16) transported from the scene of injury to a lower-level

Table 2 Variables of the prediction model incorporated in the Trauma Triage App

Age

Systolic blood pressure

Glasgow Coma Scale

Penetrating injury of head, thorax, or abdomen

Fall $>2 \mathrm{~m}$ or motorcycle accident $>30 \mathrm{~km} / \mathrm{h}$ or entrapment in vehicle

Suspected moderate or severe head injury

Suspected moderate or severe thoracic injury

Injuries in at least two anatomical regions (head/neck, face, thorax, abdomen, extremities, and/or external injuries) trauma center. This implies that on patient level, a severely injured patient can be either correctly triaged and transported to a higher-level one trauma center or incorrectly triaged and transported to a lower-level trauma center.

\section{Secondary outcomes}

- Overtriage, defined as the transportation of nonseverely injured patients (ISS < 16) from the scene of injury to a higher-level trauma center, will be evaluated on patient level.

- A non-compliance analysis will be conducted to evaluate the efficacy of the TTApp under ideal circumstances with complete adherence by all EMS professionals in the specified study population.

- Use of health care resources. A comparative analysis will be performed to evaluate the differences in hospital length of stay, number of admissions to the Intensive Care Unit, and length of stay at the Intensive Care Unit, between control and intervention conditions.

- The diagnostic accuracy of the prediction model incorporated in the TTApp will be evaluated for all eligible patients.

- A cost-effectiveness analysis will be performed alongside this SW-CRT that is described in a separate protocol.

\section{Statistical analyses Primary analysis}

The primary endpoint - undertriage - will be analyzed at a patient level using a generalized linear mixed model 
(GLMM). A random intercept will be introduced in the model to account for cluster differences. Time will be modeled as a categorical variable denoting the cluster step. The GLMM will be used in conjunction with the binomial distribution and the identity link, resulting in a risk difference between the control and intervention condition. A small sample correction will be applied owing to the small number of clusters $[9,10]$. Bootstrapped 95\% CIs will be estimated from this model. This intention-to-treat analysis will be adjusted for age, which is expected to be non-linear and thus will be modeled using restricted cubic regression splines. In addition, we will estimate relative risks and corresponding confidence intervals using a log-binomial GLMM. Missing values will be multiply imputed using a multilevel multiple imputation strategy using chained equations which accounts for cluster differences using the $\mathrm{R}$ package micemd [11]. All statistical analyses will be conducted in R (version 3.6.2; R Foundation for Statistical Computing) [12].

\section{Secondary analyses}

- Overtriage is analyzed using the same strategy as the primary analysis: a GLMM using a binomial distribution and the identity link resulting a risk difference. Bootstrapped 95\% CIs will be calculated, and analyses will be adjusted for age. All statistics are calculated for patients with an ISS $<16$.

- The primary analysis is aimed to assess the effectiveness of the TTApp. A non-compliance analysis, using instrumental variable estimation, will be conducted to evaluate the efficacy of the TTApp in the hypothetical situation with complete adherence $[13,14]$.

- Healthcare resources measured on a continuous scale (e.g., length of stay) are analyzed using a GLMM with a Gaussian distribution and the identity link. Numbers of admissions are converted to proportions and analyzed similar to the primary analysis.

- The probability generated by the logistic regression model incorporated in the TTApp will be calculated for all eligible patients based on the digital runreports and the data generated by the TTApp. Diagnostic accuracy measures, such as sensitivity, specificity, predictive values, and likelihood ratios, will be calculated and model discrimination and calibration will be assessed.

\section{Sample size}

The primary goal of the TESLA study is to evaluate whether implementation of the TTApp in daily practice reduces undertriage. The sample size calculation is based on this endpoint. The intra-cluster correlation was calculated using the Fleiss-Cuzick method on data from studies by Voskens and colleagues and the Dutch $\mathrm{Na}$ tional Trauma Registry and was 0.098 (95\% CI, 0.028$0.150)[5,6,15]$. The proportion of expected undertriage under usual care was 0.35 , whereas a decrease of 0.1 was expected during the intervention period. A decrease of 0.1 was expected based on the pre-defined threshold probability of the TTApp. With eight clusters, a power of $80 \%$, a significance level of 0.05 , and two clusters switching to the intervention after every step, at least 48 severely injured patients will have to be included per cluster per step [16, 17]. The expected power given the intra-cluster correlation interval ranges from 0.79 to 0.82 [18]. Approximately 1300-1400 severely injured patients will be transported by the participating EMSs on a yearly basis; therefore, we expect a duration of (less than) 4 months per step. Patient dropout was not expected to occur [6]. The total study time consists of a baseline period plus four additional steps, totaling 20 months in which 1920 severely injured patients should be included.

Approximately 1536 severely injured patients were included until December 1, 2019, of which 576 patients were recruited during the intervention period.

\section{Discussion}

Getting the right patient to the right hospital within a certain time frame is becoming increasingly important with the maturation of trauma systems and centralization of resources. Costs and mortality rates can be reduced by minimizing undertriage, overtriage, and interhospital transfer rates. The optimal hospital for an individual injured patient has to be determined on-scene by a diagnostic strategy that consists predominantly of (1) identification of injured patients in need of specialized trauma care and (2) logistical considerations, such as trauma center proximity and trauma center capacity. Pre-hospital triage tools, such as the Field Triage Decision Scheme, the NPAS, and the TTApp, attempt to assist EMS professionals in the first step of this strategy. These diagnostic tools should be thoroughly tested and preferably evaluated by both external validation and impact assessment before widespread implementation in clinical practice. External validation is crucial to evaluate the true performance of a prediction model in new (external) patients. Although the previously described prediction model is externally validated already, the application of the study results in practice might uncover implementation problems, disadvantages of a digital approach, and possible improvements and might thus yield different results than expected. The aim of the TESLA trial is therefore to assess the impact of the TTApp in practice. This will likely give insights into 
reasons for nonadherence, reasons to overrule the prediction model and its advice, and the isolated impact of many of the components in the diagnostic strategy that lead to the determination of the most optimal hospital.

Individual randomization of patients was not deemed appropriate during field triage, especially in time-critical settings. A stepped-wedge cluster-randomized design was chosen to mitigate logistical constraints such as labor intensity (e.g., training of EMS professionals) through phased implementation of the intervention and enabled all participating EMSs to evaluate the TTApp. Stepped-wedge cluster-randomized designs are particularly used to evaluate the impact of the implementation of prediction models in clinical practice [19]. This unidirectional crossover design combines elements of before-after studies with cluster randomization and is an efficient design that enables to derive a valid answer for the research question.

This study is limited by the fact that current population values were used to determine the sample size. These values might not reflect actual event rates during the trial. Patients require an ISS $\geq 9$ to be eligible for inclusion in this trial. This is based on the assumption that EMS professionals are able to differentiate between mildly injured patients and those who are severely injured, which is likely, but might not entirely resemble actual usage. This constraint was posed to limit overtriage of a clearly non-severely injured group of patients. A second limitation is the use of an exchangeability correlation structure in the analyses. Since the design of this study, methodological literature has been moving towards more complex correlation structures (e.g., discrete time decay correlation) which might be more appropriate for stepped-wedge study designs [18]. Another potential limitation of the trial is the innovativeness of the TTApp and subsequently its dissimilarity to routinely used static decision schemes. This could potentially lead to substantial nonadherence. Due to these reasons and because of the fixed length of the steps in a SW-CRT and its inextensible nature, the sample size was calculated with a conservative estimate of the expected decrease in undertriage.

\section{Conclusion}

The TESLA trial is a SW-CRT that aims to evaluate the impact of the TTApp on the primary endpoint undertriage, as well as overtriage and hospital resource use. The smartphone application can potentially acquire new and previously undiscovered insights into several components of the strategy that leads to the determination of the optimal hospital for a specific injured patient.

\section{Abbreviations}

EMS: Emergency Medical Services; NPAS: National Protocol of Ambulance Services; TTApp: Trauma Triage App; TESLA: Trauma Triage using Supervised
Learning Algorithms; SW-CRT: Stepped-wedge cluster-randomized trial; ISS: Injury Severity Score; GLMM: Generalized linear mixed model

\section{Acknowledgements}

Not applicable.

\section{Authors' contributions}

RvdS contributed to the study design, wrote the statistical analysis plan, and drafted and revised the paper. AAAF, JFW, JBR, LPHL, MvH, and MP contributed to the study design, analysis plan, and revision of the paper. All authors were involved in the study design and revised the draft paper. The authors read and approved the final manuscript.

\section{Funding}

This study is financially supported by The Netherlands Organization for Health Research and Development, Effectiveness Program (ZonMw [https:// www.zonmw.nl/en/], dossier number 80-84300-98-18555). The TESLA-trial is an investigator-initiated trial. The funding body has no role in the design of the study, collection, analysis, and interpretation of data and neither in writing the manuscript.

\section{Availability of data and materials}

Study results will be disseminated via a peer-reviewed international medical journal. Designated authors have to meet all four criteria for authorship in the International Committee of Medical Journal Editors (ICMJE) recommendations.

\section{Ethics approval and consent to participate}

The study is performed in accordance with the declaration of Helsinki and the Dutch Medical Research Involving Human Subjects Act. Referring to the approval letter from the IRB of the Zuyderland Medical Center (Limburg, the Netherlands) on May 23, 2018 (reference number METCZ20180069), it is confirmed that the Medical Research Involving Human Subjects (WMO) does not apply to the above-mentioned study. Written consent to participate was obtained for every participating EMS and their healthcare workers, by means of the medical manager of ambulance care.

\section{Consent for publication}

No identifying images or other personal or clinical details of participants are presented that compromise anonymity.

\section{Competing interests}

The authors declare that they have no competing interests.

\section{Author details}

${ }^{1}$ Department of Surgery, Maastricht University Medical Center, Maastricht, The Netherlands. ${ }^{2}$ Department of Surgery, Utrecht University Medical Center, Utrecht, The Netherlands. ${ }^{3}$ Network Acute Care Limburg, Maastricht University Medical Center, Maastricht, The Netherlands. ${ }^{4}$ Department of Epidemiology, Julius Center, University Medical Center Utrecht, Utrecht, The Netherlands. ${ }^{5}$ Department of Surgery, Erasmus Medical Center, Rotterdam, The Netherlands. ${ }^{6}$ Care and Public Health Research Institute, Maastricht University, Maastricht, The Netherlands. ${ }^{7}$ Department of Surgery, Amsterdam University Medical Center, Amsterdam, The Netherlands. ${ }^{8}$ Department of Surgery, Onze Lieve Vrouwe Hospital, Amsterdam, The Netherlands.

${ }^{9}$ Ambulance Care the Netherlands, Zwolle, The Netherlands. ${ }^{10}$ Department of Surgery, Elisabeth TweeSteden Hospital, Tilburg, The Netherlands.

${ }^{11}$ Department of Surgery, Diakonessenhuis Utrecht/Zeist/Doorn, Utrecht, The Netherlands.

Received: 7 January 2020 Accepted: 22 April 2020

Published online: 18 June 2020

\section{References}

1. American College of Surgeons Committee on Trauma. Resources for optimal care of the injured patient 2014: Chicago, IL; 2014. https://www.facs. org/quality-programs/trauma/tqp/center-programs/vrc/resources (accessed Aug 20, 2019).

2. Mackenzie EJ, Rivara FP, Jurkovich GJ, Nathens AB, Frey KP, Egleston BL, Salkever DS, Scharfstein DO. A national evaluation of the effect of traumacenter care on mortality. N Engl J Med. 2006;354(4):366-78. 
3. Newgard CD, Staudenmayer K, Hsia RY, Mann NC, Bulger EM, Holmes JF, Fleischman R, Gorman K, Haukoos J, McConnell KJ. The cost of overtriage: more than one-third of low-risk injured patients were taken to major trauma centers. Health Aff (Millwood). 2013;32(9):1591-9.

4. Dutch National Healthcare Institute: Spoed moet goed: indicatoren en normen voor zes spoedzorgindicaties; 2015.

5. Landelijk Netwerk Acute Zorg: Landelijke Traumaregistratie 2012-2016. Utrecht, 2017.

6. Voskens FJ, van Rein EAJ, van der Sluijs R, et al. Accuracy of pre-hospital triage in selecting severely injured trauma patients. JAMA surgery. 2018; 153(4):322-7.

7. van Rein EAJ, Houwert RM, Gunning AC, Lichtveld RA, Leenen LPH, van Heijl M. Accuracy of prehospital triage protocols in selecting severely injured patients: a systematic review. J Trauma Acute Care Surg. 2017;83(2):328-39.

8. Van Rein EAJ, van der Sluijs R, Voskens FJ, Dijkgraaf M, Lichtveld RA, Houwert RM, Leenen LP, van Heijl M. A prediction model as basis for prehospital triage of trauma patients in the Netherlands. JAMA Surg. 2019.

9. Barker D, D'Este C, Campbell MJ, McElduff P. Minimum number of clusters and comparison of analysis methods for cross sectional stepped wedge cluster randomised trials with binary outcomes: a simulation study. Trials. 2017;18(1):119.

10. Li P, Redden DT. Comparing denominator degrees of freedom approximations for the generalized linear mixed model in analyzing binary outcome in small sample cluster-randomized trials. BMC Med Res Methodol. 2015;15:38.

11. Audigier V, Resche-Rigon M: micemd: multiple imputation by chained equations with multilevel data. Version 1.6.0; 2019.

12. R Core Team: R: a language and environment for statistical computing. Vienna, Austria: R Foundation for Statistical Computing; 2019.

13. Hernan MA, Hernandez-Diaz S. Beyond the intention-to-treat in comparative effectiveness research. Clin Trials. 2012;9(1):48-55.

14. Toh S, Hernan MA. Causal inference from longitudinal studies with baseline randomization. Int J Biostat. 2008:4(1):22

15. WU S, Crespi CM, Wong WK. Comparison of methods for estimating the intraclass correlation coefficient for binary responses in cancer prevention cluster randomized trials. Contemp Clin Trials. 2012;33(5):869-80.

16. Hooper R, Bourke L. Cluster randomised trials with repeated cross sections: alternatives to parallel group designs. BMJ. 2015;350:h2925.

17. Hooper R, Teerenstra S, de Hoop E, Eldridge S. Sample size calculation for stepped wedge and other longitudinal cluster randomised trials. Stat Med. 2016;35(26):4718-28.

18. Hemming K, Kasza J, Hooper R, Forbes A, Taljaard M. A tutorial on sample size calculation for multiple-period cluster randomized parallel, cross-over and stepped-wedge trials using the Shiny CRT Calculator. Int J Epidemiol. 2020.

19. Mdege ND, Man MS, Taylor Nee Brown CA, Torgerson DJ. Systematic review of stepped wedge cluster randomized trials shows that design is particularly used to evaluate interventions during routine implementation. J Clin Epidemiol. 2011;64(9):936-48.

\section{Publisher's Note}

Springer Nature remains neutral with regard to jurisdictional claims in published maps and institutional affiliations.

Ready to submit your research? Choose BMC and benefit from:

- fast, convenient online submission

- thorough peer review by experienced researchers in your field

- rapid publication on acceptance

- support for research data, including large and complex data types

- gold Open Access which fosters wider collaboration and increased citations

- maximum visibility for your research: over $100 \mathrm{M}$ website views per year

At $\mathrm{BMC}$, research is always in progress.

Learn more biomedcentral.com/submissions 